
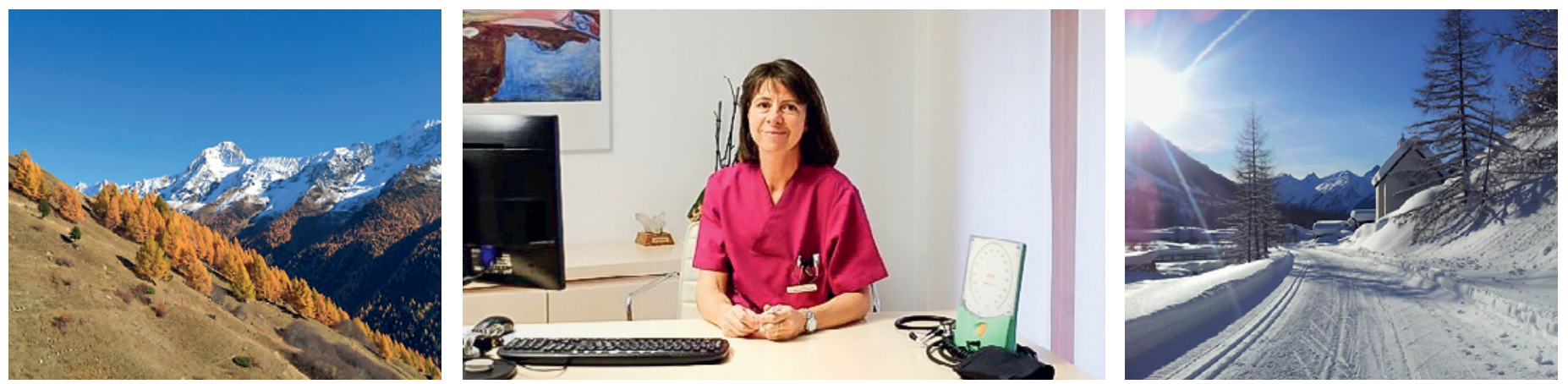

Lutgard Werlen stammt aus Belgien und ist seit mehr als zwölf Jahren Talärztin im Lötschental (Wallis).

\title{
"Niemand hat gedacht, dass ich bleiben werde»
}

\section{Interview mit Lutgard Werlen: Tanja Kühnle}

Rund ein Drittel der Ärztinnen und Ärzte in der Schweiz kommt aus dem Ausland. Weshalb haben sie sich für ein Leben hierzulande entschieden? Welches sind kulturelle Stolperfallen, die es zu umschiffen gilt? Und was halten sie von der Schweizer Gesundheitspolitik? Diesen und weiteren Fragen möchten wir in der in loser Folge erscheinenden Artikelserie «Grüezi Schweiz» nachgehen. In dieser Ausgabe erzählt uns Talärztin Lutgard Werlen, wie sie den Weg ins Lötschental fand.

\section{Der Weg in die Schweiz}

Lutgard Werlen ist, wie sie selbst sagt, schon während ihres Medizinstudiums in der Schweiz «hängen geblieben». Sie war 17 Jahr alt und hatte gerade erst das Medizinstudium in ihrer Heimat Belgien begonnen, als sie im Wallis Urlaub machte und sich in ihren Bergführer verliebte. Nach sechs Jahren Fernbeziehung - sie wollte erst das Medizinstudium in Gent beenden - läuteten dann die Hochzeitsglocken, und sie zog 1997 zu ihrem Mann ins Lötschental.

In dieser abgeschiedenen Region sind der Pfarrer und der Arzt noch sehr wichtig.

Was sie dort genau erwartete, wusste sie nicht. «Ich habe das Lötschental gut gekannt. Aber ich kam aus der Stadt. Und bin aufs Land, in die Berge, gezogen. Das Leben hier ist ganz anders.» In dieser abgeschiedenen
Region seien der Pfarrer und der Arzt noch sehr wichtig, sagt sie. Und das findet sie schön. Auch das Klima sei besser, die Natur schöner. Und die Arbeit vielfältiger als daheim. Dazu später mehr.

\section{Vom Spital in die Praxis}

Gearbeitet hat Lutgard Werlen zunächst im Spital in Brig. Sie war damals die erste ausländische Ärztin auf der inneren Medizin. Nach einiger Zeit träumte sie davon, im Lötschental eine eigene Praxis für Allgemeinmedizin zu eröffnen. Nachdem der letzte Talarzt das Tal verlassen hatte, realisierte sie 2006 ihren Traum. Seither ist sie Talärztin mit Leidenschaft und rund um die Uhr für ihre Patienten da.

Rund um die Uhr? Ist es nicht sehr stressig, immer im Dienst zu sein? «Es geht», sagt Lutgard Werlen. «Ich kann gut abstellen, und wenn ein Anruf kommt, dann bin ich einsatzbereit.» Ihre Kinder waren früher jedoch 
nicht davon begeistert, dass die Mama so häufig wegmusste. «Sie haben mich oft gebeten, dass ich doch einfach sagen soll, dass ich keine Zeit habe. Doch das kam für mich nicht in Frage. Und mittlerweile haben sie sich daran gewöhnt.»

\section{Kulturelle Stolperfallen?}

Damit hatte Lutgard Werlen nie zu kämpfen. Und Anschluss zu finden, war für sie auch kein Problem. Zum einen vermutlich, weil sie einen Einheimischen geheiratet hat. Zum anderen, weil sie sich sehr bemühte, sich zu integrieren. «Man sollte sich anpassen, wenn man

\section{Da der Lötschentaler Dialekt sehr eigen ist, hat ihn Lutgard Werlen ganz zu Beginn sogar ein wenig studiert.}

in ein Land kommt», findet die Medizinerin. "Insbesondere sollte man versuchen, den Dialekt zu sprechen. Oder ihn zumindest verstehen lernen. Das ist ganz besonders wichtig, wenn man Arzt in einer Allgemeinpraxis ist - schliesslich muss man seine Patienten verstehen können.» Und da der Lötschentaler Dialekt sehr eigen ist, hat ihn Lutgard Werlen ganz zu Beginn sogar ein wenig studiert. Mittlerweile spricht sie ihn deshalb so gut, dass Fremde nicht mehr merken, dass sie keine Einheimische ist.

\section{Belgien vs. Schweiz - was ist anders?}

"Als ich herkam, fiel mir gleich auf, dass man die Rezepte hier anders kontrolliert als in Belgien", sagt Lutgard Werlen. In Belgien habe man nummerierte Rezepte mit einem Strichcode. So liesse sich überprüfen, welcher Arzt was verschreibt. "Aber ob das notwendig oder besser ist? Ich weiss es nicht.» Ansonsten sei das System sehr ähnlich, und sie habe sich nicht sonderlich umstellen müssen. Allerdings sei ihre Arbeit in der Schweiz als Allgemeinmedizinerin vielfältiger. Denn in Belgien dürfe man als Hausarzt kein eigenes Labor haben, keine Röntgenaufnahmen machen und auch keine MPA einstellen. «Bei uns in Belgien gibt es nur eine Sekretärin, die das Telefon entgegennimmt. Hier darf ich viel mehr machen und das gefällt mir sehr.»

\section{Und das Schweizer Gesundheitssystem?}

«Ich finde, die Schweizer Gesundheitsversorgung ist sehr gut. Und die Qualität der Medizin extrem hoch. Man kann alle möglichen Untersuchungen haben - meistens sogar ohne Wartezeit. In England ist das anders. Ich habe dort mal ein halbes Jahr gearbeitet und die Erfahrung gemacht, dass Spezialgeräte wie CT oder MR weniger zugänglich sind als hier.» In der Schweiz führe diese hohe Verfügbarkeit ihrer Erfahrung nach aber auch dazu, dass viele Patienten Untersuchungen fordern, die gar nicht notwendig seien. Nach dem Motto: Mein Kollege hatte bei ähnlichen Beschwerden ein MR bekommen, also möchte ich das jetzt auch. «Als ich angefangen habe, im Wallis zu arbeiten, gab es ein MR in Sitten. Jetzt gibt es welche in allen Städten - und alle sind ausgelastet. Ob diese Untersuchungen alle nötig sind?»

\section{Wünsche für die Zukunft}

"Ich bin sehr zufrieden und hoffe, dass das Gesundheitssystem weiterhin so funktioniert wie bisher.» Aber auch Lutgard Werlen wird nicht jünger. Und deshalb fragt sich die Ärztin manchmal, ob sie ihren Beruf auch noch in einigen Jahren so ausüben kann wie bisher. Nach Belgien zurückzugehen sei keine Option. «Ich wüsste nicht, warum. Ich bin jetzt schon fast genauso lange hier, wie ich in Belgien gelebt habe.»

\section{Die Schweiz, ein Traumarbeitsort für Ärztinnen und Ärzte?}

Lutgard Werlen mag ihr Leben im Lötschental. «Ich bin sehr zufrieden hier. Es gibt nichts, was mich stört oder was ich ändern möchte. Ich mag meine Arbeit, die Berge und den Sport. Aber ob ich das Leben hier anderen empfehlen soll, weiss ich nicht.» Schliesslich sei es nicht jedermanns Sache, allein eine Talpraxis zu führen und immer zu 100\% präsent zu sein. Und zwei

Ich mag meine Arbeit, die Berge und den Sport.

Vollzeitstellen gäbe die Arbeit nicht her. «Ich denke, dass die Zukunft auch hier eher in Richtung Gruppenpraxis geht. Vielleicht werde ich irgendwann nicht einen, sondern zwei Nachfolger haben, die sich die Arbeit teilen.»

Bildnachweis

Fotos zur Verfügung gestellt von Lutgard Werlen

Für unsere Serie "Grüezi Schweiz» suchen wir ausländische Ärztinnen und Ärzte, die uns einen Einblick in ihr Leben und ihren Berufsalltag gewähren. Wir freuen uns über eine Kontaktaufnahme: tkuehnle[at]emh.ch 